\title{
El peso del apego y de la cultura en las estrategias de rompimiento amoroso percibidas por los abandonados
}

\author{
Blanca Estela Retana-Franco; Rozzana Sánchez-Aragón
}

How to cite this article:

Retana-Franco, B.E., \& Sánchez-Aragón, R. (2020). The impact of emotional attachment and culture on the breakup strategies perceived by the abandoned. Acta Colombiana de Psicología, 23(1), 53-65. doi: http://www.doi.org/10.14718/ ACP.2020.23.1.4

Recibido, noviembre 7/2018; Concepto de evaluación, abril 1/2019; Aceptado, abril 11/2019

\author{
Blanca Estela Retana-Franco \\ Universidad Pedagógica Nacional, Ciudad de México, México \\ ORCID: https://orcid.org/0000-0002-1393-3665 \\ Rozzana Sánchez-Aragón \\ Universidad Nacional Autónoma de México, Ciudad de México, México \\ ORCID: https://orcid.org/0000-0002-5952-8972
}

\begin{abstract}
Resumen
Dada la importancia que se confiere a las relaciones de pareja, su rompimiento generalmente repercute de manera negativa en la vida de sus miembros, sobre todo en quien es víctima de la decisión del otro - es decir, quien ejerce un papel pasivo-. Así, desde el punto de vista cultural, cuando se le atribuye a la relación de pareja un valor importante como fuente de realización personal y familiar, y ello se transmite a través de los miembros - lo que facilita el desarrollo de creencias, normas, actitudes y expectativas del amor- - su búsqueda y consumación se vuelve una directriz en la vida de las personas. Adicional a esto, si el apego — que representa el medio por el cual se cubren las necesidades biológicas de afecto y seguridad- se ve satisfecho en la vinculación con el otro, puede que resulte devastador perder la fuente de tales satisfactores. Con base en lo anterior, el propósito de este estudio fue identificar la relación entre los estilos de apego y las premisas histórico-socioculturales (PHSC) del duelo romántico con las estrategias de rompimiento percibidas por los abandonados con historia romántica positiva o negativa. Para ello, se trabajó con una muestra no probabilística de 547 personas residentes de la Ciudad de México que habían sido dejadas por su pareja. Tras realizar algunos análisis de correlación, los resultados indicaron que las PHSC tienen mayores efectos sobre la percepción de estrategias de rompimiento negativo tanto en personas con historia romántica positiva como negativa. Los efectos incrementan cuando se trata de las PHSC sobre el sentir, principalmente en abandonados con historia negativa y con estilo de apego ansioso-ambivalente, seguido del evitativo. Por su parte, las PHSC positivas se asocian, pero en un nivel bajo, con cualquier estrategia percibida.

Palabras clave: apego, cultura, rompimiento amoroso, abandonados, México.
\end{abstract}

\section{The impact of emotional attachment and culture on the breakup strategies perceived by the abandoned}

\begin{abstract}
Considering the importance that has it been given to couple relationships, their breakdown has a negative impact on its members, especially on those who are victims (passive role) of the other's decision. Thus, from the cultural point of view, when a great value is attributed to romantic relationships, in terms of personal and family fulfillment, and this is transmitted to the members of the couple, facilitating the development of beliefs, norms, attitudes and expectations about the love experience, the search and consummation of that love becomes a guideline in people's lives. And if coupled with this, the attachment representing the means by which the biological needs for affection and security are met through the connection with another, it can be devastating to lose the source of such satisfactions. Based on the foregoing, the purpose of this study was to identify the relationship between the historic socio-cultural premises (PHSC, for its Spanish acronym) about
\end{abstract}

Calzada Azcapotzalco, La Villa, n. ${ }^{\circ}$ 1011, San Andrés de las Salinas, C. P.: 02320, Azcapotzalco, Ciudad de México.bretana@upn.mx 
romantic mourning and the attachment styles with the breakup strategies perceived by the passive subjects with a positive and negative romantic history. To do this, the study used a non-probabilistic sample of 547 residents of Mexico City who had been left by their partner. After performing some correlation analyzes, results indicated that the PHSC's have greater effects on the perception of negative breakup strategies in people with a positive or negative romantic history. The effects increased when dealing with PHSC's about feelings, mainly in passive members with a negative history and with an anxious attachment style followed by those with avoidant style. For their part, positive PHSCs are associated, but at a low level, with any perceived strategy.

Key words: attachment, culture, breakup, bandoned, Mexico.

\section{Introducción}

El establecimiento de relaciones afectivas resulta de mayor importancia en ciertas etapas de la vida del individuo; tal es el caso de la población joven adulta, pues el hecho de elegir una carrera profesional que dé un sentido de vida, recursos y la posibilidad de crecimiento personal, así como iniciar una relación e ir aprendiendo a vivir en compañía, terminan siendo situaciones trascendentales para su desarrollo personal (Rage, 2002). Teniendo esto en cuenta, las relaciones afectivas de pareja representan un aspecto fundamental en la vida del individuo (Becerril, 2001), y en ellas la comunicación y la comprensión son esenciales para el adecuado desarrollo de cada persona y de su vida en sociedad. Asimismo, dado que estas relaciones son las más íntimas, de igual forma son las que dan un soporte significativo al individuo a niveles tanto afectivos como sociales. Sin embargo, ¿qué pasa cuando estas relaciones de pareja no funcionan?

Cuando acaece la ruptura, en la que se tiene la experiencia de un estresor doloroso que provoca el derrumbe de perspectivas y aspiraciones, así como la modificación de aspectos personales, familiares, económicos y sociales, emerge un ambiente percibido como desalentador y frustrante para quienes hacían parte de la pareja (Jaramillo-Bustamante, 2015). Según Caruso (2003), el rompimiento amoroso es una de las experiencias más dolorosas para el ser humano, $\mathrm{y}$ aunque puede variar en intensidad, dependiendo de los factores que intervienen en ella, si no se maneja adecuadamente, puede llegar a tener consecuencias relativamente graves, que pueden ir desde la tristeza y el enojo hasta desórdenes psicológicos como la ansiedad y la depresión (Fisher, 2005).

En la literatura resaltan algunos datos importantes al respecto, como lo son: (a) que la cultura en la cual se nace posee una serie de normas y creencias respecto a lo que es el amor, así como de su valor, los mecanismos de funcionamiento de las relaciones y lo que trae consigo su pérdida (Díaz-Loving \& Sánchez-Aragón, 1998; SánchezAragón, 2018); (b) que el estilo de apego determina, en cierta medida, cómo, cuándo, por qué y con quién se relacionará una persona afectivamente en el futuro (Le, Dove, Agnew, Korn \& Mutso, 2010); (c) que la historia amorosa de cada persona predispondrá la experiencia y proceso del duelo; y (d) que la estrategia que se ejecute para dar por terminada la relación también se ve influenciada por los factores mencionados (Collins \& Gillath, 2012; Jiménez-Rodríguez \& Sánchez-Aragón, 2017).

Es importante recordar que las relaciones y los vínculos no se establecen en el espacio vacío, sino que se rigen por las normas o preceptos culturales donde se desenvuelve la relación amorosa. Teniendo esto en cuenta, la sociocultura - definida por Díaz-Guerrero (2003) como un sistema de creencias interrelacionadas y generadas a partir de la cultura y la sociedad - cumple el papel de regular los sentimientos, las ideas, la jerarquización de las relaciones interpersonales y la estipulación de los papeles sociales, así como de establecer las reglas de interacción para los individuos que cumplen tales papeles.

Según Díaz-Guerrero (2003), estos mecanismos de regulación son representados por las premisas históricosocioculturales (PHSC), que hacen referencia a un sistema de creencias y valores que actúan como normas o mandatos que estipulan los roles de los individuos, las prácticas sociales y los estilos de confrontación de una socio-cultura. Así, cuando los miembros de una sociedad crecen creyendo que el amor romántico y la pareja son el cimiento de la familia y que a través de ella se logra la realización personal (Guttmann, 1993), el rompimiento amoroso, y su consecuencia legal, el divorcio —o la separación-, se ven como una decisión equivocada, como algo personal, y no como una falla socioestructural que presiona a las personas a tratar una y otra vez de embarcarse en la búsqueda de una nueva y más compatible pareja (Feldberg \& Kohen, 1980). Es de esta manera como la socio-cultura moldea las experiencias positivas y negativas inherentes al amor romántico, incluido también el duelo por rompimiento amoroso.

En México, Díaz-Loving y Sánchez-Aragón (1998) identificaron una serie de PHSC comunes de la pareja mexicana relativas al conflicto y la separación, la pasión, 
el amor de compañía, el compromiso, el mantenimiento, el romance, la tristeza, el amor trágico, el desamor, el alejamiento, y, por último, la atracción. Por dar un ejemplo, en cuanto al contenido de las premisas relativas al proceso de duelo romántico, los autores identificaron oraciones como "cuando una relación se torna insoportable se debe dar la separación", "evadir a la pareja es señal de alejamiento", "cuando la pareja se aleja es porque ya se perdió el interés en la relación", "la separación de la pareja produce tristeza y depresión", "cuando se ha perdido un amor se sufre", "durante la separación de la pareja hay soledad", "cuando las parejas se alejan es porque ya no se aman".

Siguiendo esta dirección, Sánchez-Aragón (2018) realizó un estudio exploratorio y diseñó una medida de PHSC para el duelo romántico en la que se aborda ampliamente la manera en que los mexicanos sienten, piensan y actúan ante el rompimiento, en donde emergieron aspectos como, respecto al pensamiento, pensar positivo, en los motivos, en la reconciliación, y pensar con fatalismo; frente al sentimiento, sentirse devaluado, melancólico, terrible y con resentimiento; $y$, con respecto a las conductas, comportarse con fortaleza, en paz, animarse y buscar alternativas.

Con estos dos estudios, solo por mencionar algunos, se hace evidente el peso que tiene la cultura en la manera en la que se experimenta el duelo romántico, pues los valores culturales matizan las creencias y establecen las reglas de interpretación y de comportamiento apropiado ante una situación de crisis personal y relacional en determinado grupo cultural.

Por otra parte, también es importante mencionar que, aunado a la cultura, el estilo de apego que cada persona desarrolla a partir de su vinculación con su cuidador primario $\mathrm{y}$, en consecuencia, con otras personas significativas a lo largo de su vida romántica, tiene un gran impacto en la experiencia del duelo romántico (Le et al., 2010).

Específicamente, el apego ha sido definido como una especie de "lazo psicológico" producto de las sensaciones agradables y desagradables que se experimentan al lado de una persona significativa - en el recién nacido, su cuidador primario; posteriormente, otras personas - de quien se espera tener acceso para satisfacer necesidades de atención, cuidado y afecto (Ainsworth, 1969; Bowlby, 1973), y con quien se busca compartir, sentir confort, tener cercanía y disfrutar de su compañía en términos de significado y sentido para la vida personal (Bowlby, 1973). Dependiendo de las interacciones del infante con su cuidador primario y de la eficacia de este como figura de apego - en su respuesta a las necesidades y demandas del otro- es que se desarrollan los diferentes patrones o estilos de relacionamiento que, en un futuro, predispondrán a las personas a vincularse con otros (Bartholomew \& Horowitz, 1991), y, para el caso de la presente investigación, con la pareja (Hazan \& Shaver, 1987).

Desde esta perspectiva, los estilos de apego mejor identificados son:

1. Estilo seguro: propio de una persona cuya figura de apego ha respondido adecuadamente y en el momento preciso a sus necesidades, lo cual repercute en una sensación de estar protegido, tranquilo, lleno de confianza y sin autopercepción de vulnerabilidad. Con estas características, ante una eventual separación, el comportamiento de un individuo está libre de miedo y de ansiedad, se mantiene la calma y, frente a la presencia y ausencia de la figura de apego, el sujeto realiza comportamientos que expresan accesibilidad, certidumbre y cuidado (Bowlby, 1973). Estos individuos pueden gravitar hacia el desarrollo estable de sus relaciones interpersonales, pues soportan relativamente altos niveles de confianza, interdependencia, compromiso y satisfacción (Simpson, 1990), y porque tienden a ser tolerantes ante los desacuerdos con el otro miembro de la relación (Remshard, 1998).

2. Estilo ansioso-ambivalente: caracterizado por la carencia de respuesta precisa y constante por parte de la figura de apego, lo cual favorece duda e inseguridad persistentes frente a tener acceso a esta persona, así como una sensación de inquietud permanente. En este caso, ante una eventual separación, la persona suele expresar en sus comportamientos angustia excesiva, poca concentración en la tarea que realiza, e, incluso, sentimientos de nostalgia; cuando la fuente de apego regresa, expresa falta de confianza y conductas de enojo y protesta (Tzeng, 1992).

3. Estilo evitativo: se caracteriza por la permanente inaccesibilidad a la figura de apego para satisfacer las necesidades tanto de atención y cuidado como de interpretación emocional a los estímulos que se experimentan como estresantes, dolorosos y de rechazo. En este caso, ante una separación, la persona con este estilo suele manifestar angustia, pasividad, tristeza, o exhibir un aparente desinterés e inseguridad hacia los demás, por lo cual prefiere mantener distancia de los otros; estos individuos también suelen tener miedo a la intimidad y muestran dificultades para depender de las otras personas (Mikulincer \& Shaver, 2003). La diferencia con este estilo de apego es que no existe un reclamo de ausencia hacia la figura de apego, pues cuando esta regresa, simplemente evita el contacto y la rechaza como una forma de responder defensivamente y de autoprotegerse (Tzeng, 1992). Asimismo, estas personas tienden a desarrollar modelos mentales suspicaces, lejanos y de escepticismo, por lo que sus relaciones llegan a ser muy pobres. En cuanto a la intensidad y profundidad de su nivel de intimidad, tienden a desarrollar modelos mentales de miedo, desconfianza y sobreapreciación - un tanto 
irreal para los otros-, y son incapaces de entregarse a una relación permanente (Fuller \& Fincham, 1995).

Ahora bien, en el escenario del rompimiento amoroso, Davis, Shaver y Vernon (2003) analizaron los estilos de apego en adultos jóvenes estadounidenses que habían atravesado por dicha situación, $\mathrm{y}$ encontraron que los participantes con un estilo de apego ansioso-ambivalente tenían una mayor dificultad para superar dicha experiencia, mientras que quienes tenían un estilo de apego seguro eran más propensos a buscar apoyo social para hacer frente a dicha pérdida. Por su parte, Sbarra (2006) confirmó estos hallazgos al reportar que para los participantes con un estilo de apego ansioso-ambivalente fue mucho más difícil superar la tristeza de la pérdida y aceptar que la relación había terminado, además de que, al momento del estudio, los individuos con este estilo de apego continuaban sumamente enamorados de su expareja, a la vez que manifestaban fuertes sentimientos de enojo hacia ella.

Adicional a esto, se ha identificado la existencia de correlaciones entre patrones de unión y rupturas, por ejemplo, la asociación positiva entre el apego ansioso-ambivalente y la preocupación por la ruptura, la angustia física y emocional y el comportamiento de enojo y venganza después de una ruptura; o entre la ansiedad y el número de intentos para restablecer la relación y más comportamiento de búsqueda no deseado hacia la expareja - lo que lleva a un círculo vicioso- (Dutton \& Winstead, 2006). También, el apego evitativo se ha asociado a una mayor tendencia a la ruptura, a reacciones emocionales más débiles y a menos conductas de búsqueda de proximidad después de que ocurre (Feeney \& Noller, 1992); mientras que la tendencia de los individuos con alto grado de evitación es a evadir la intimidad y las confrontaciones potenciales con su pareja (Schachner \& Shaver, 2002). Incluso, se ha encontrado una asociación entre las personas más seguras - con baja ansiedad y evitación- y el hecho de experimentar menos rupturas, usar estrategias de afrontamiento más sociales y menos autodestructivas, y una mayor probabilidad de llegar a una resolución después de la ruptura (Davis et al., 2003). Estos resultados, junto con la literatura sobre patrones de apego $\mathrm{y}$ tendencias prosociales, sugieren que las dimensiones de apego podrían predecir el tipo de estrategias de ruptura que las personas prefieren.

Justamente, estos hallazgos ejemplifican a la perfección el hecho de que una persona que cuenta con cierto tipo de apego está hasta cierto punto "predestinada" a experimentar, en forma reiterativa, cierto tipo de emociones y pensamientos con respecto a sus relaciones en el transcurso de su vida, lo cual define su historia amorosa en términos positivos o negativos. Asimismo, el estilo de apego determinaría en la persona su percepción e interpretación de lo relativo a las circunstancias que rodean el rompimiento amoroso.
Por otra parte, aunado a los factores previamente mencionados, la literatura señala que en la experiencia del rompimiento amoroso resulta clave considerar a la persona que tomó la decisión de la ruptura, ya que, si la decisión fue tomada por ambos miembros de la pareja, el proceso de pérdida es menos desconcertante o intenso que en situaciones en donde la decisión del rompimiento es unilateral, ya sea por parte de quien dio por terminada la relación - agente - o por quien tuvo que aceptar la decisión del otro - pasivo- (Agnew, 2000).

Al respecto, Tashiro y Frazier (2003) estudiaron las diferencias en los niveles de malestar emocional experimentado tanto en las personas que iniciaron la ruptura como en aquellas que terminaron una relación en contra de su voluntad, pero no encontraron diferencias significativas; resultados que coinciden con lo reportado por Waller (2008) y Locker, McIntosh, Hackney, Wilson y Wiegand (2010), pero no con los hallazgos de Perilloux y Buss (2008), pues en estos se encontró que los participantes que habían pasado por una ruptura amorosa en contra de su voluntad experimentaron más sentimientos de tristeza, enojo, confusión y celos después de una ruptura, además de mayor depresión, menor autoestima y más pensamientos intrusivos en comparación con aquellos que iniciaron la separación. Asimismo, Lewandowski y Bizzoco (2007) encontraron más emociones positivas y menos negativas en las personas que habían iniciado la separación que quienes reportaron ser el blanco de la ruptura.

Por último, con respecto a las estrategias que las personas utilizan para comunicar su mensaje de ruptura, Banks, Altendorf, Greene y Cody (1987) indican que las personas pueden vivir distintos grados de angustia dependiendo de la forma en que se dé la ruptura. En particular, dichas estrategias tienden a variar en su nivel de franqueza y en la cantidad de cuidado o preocupación expresada hacia la persona que ejerce el papel pasivo (Sprecher, Zimmerman \& Abrahams, 2010), ya que, por ejemplo, las estrategias que implican la comunicación indirecta del mensaje de ruptura con el compañero - como evitar a la otra personareflejan menos compasión por el receptor y se relacionan con un mayor número de resultados negativos posteriores a la ruptura (Metts, Cupach \& Bejlovec, 1989; Sprecher et al., 2010); mientras que las estrategias de ruptura que son más sinceras - como expresar abiertamente el deseo de ruptura - reflejan la mayor compasión o preocupación por la pareja y correlacionan con menos resultados negativos (Sprecher et al., 2010).

Con base en lo anterior, y teniendo en cuenta la importancia de indagar en un solo planteamiento con el fin de que se puedan vincular estas variables, el propósito de la presente investigación fue identificar la relación existente 
entre las PHSC del duelo romántico, los estilos de apego y las estrategias de rompimiento percibidas por los abandonados con una historia romántica positiva o negativa.

\section{Método}

\section{Participantes}

Se trabajó con una muestra no probabilística propositiva (Kerlinger \& Lee, 2002) de 547 participantes que reportaron haber terminado su relación de pareja por decisión de la otra persona - es decir, que ejercían un papel pasivo en la ruptura- - estar viviendo un duelo romántico. De ellos, se contó con 262 hombres y 285 mujeres con edades entre los 14 y los 48 años $(M=20.03, D E=4.22)$. En cuanto a su escolaridad, la mayoría tenía estudios de preparatoria $(38.7 \%)$ o licenciatura $(59.7 \%)$, y, con respecto a su relación romántica, los participantes reportaron que, en promedio, esta había durado alrededor de 16.11 meses y que había sido finalizada hace 5.61 meses. Del total de los participantes, cuando se les preguntó "¿cómo diría usted que ha sido su historia de relaciones románticas?", 380 señalaron haber tenido una historia romántica positiva, y 160 una negativa.

\section{Tipo de estudio}

La presente investigación consistió en un estudio correlacional y cuantitativo (Kerlinger \& Lee, 2002), debido a que se midió el grado de relación entre más de dos variables: el apego, las premisas histórico-socioculturales del duelo romántico $\mathrm{y}$ las estrategias de rompimiento amoroso en abandonados.

\section{Instrumentos}

Escala de premisas histórico-socioculturales del duelo romántico (Sánchez-Aragón, 2018). Esta escala se compone de tres subescalas que evalúan la forma en la que una persona que pasa por un duelo romántico debe pensar — subescala cognoscitiva, 31 reactivos - , sentir - subescala afectiva, 27 reactivos - y actuar -subescala conductual, 26 reactivos-. Todos los reactivos tienen un formato de respuesta tipo Likert de cinco puntos que indican grados de acuerdo o desacuerdo, con los cuales, dependiendo de la subescala, los participantes responden a un estímulo como "cuando las personas experimentan un rompimiento amoroso deben... pensar/sentir/hacer...".

En particular, la subescala cognoscitiva incluye cinco factores, a saber: (a) optimismo $(\alpha=.84 ; .81)$, con ítems como ...debe (pensar)... "en superarlo", "que hay que seguir adelante" o "positivo"; (b) alternativas y aceptación $(\alpha=.79 ; .88)$, con reactivos como ...debe (pensar)... "que hay mejores personas en el mundo", "quizá él/ella no era la mejor persona para él/ella" o "que existen mejores oportunidades en la vida"; (c) motivos ( $\alpha=.73 ; .74)$, con enunciados como ...debe (pensar)... "en los motivos que dieron pie al rompimiento", "sobre las razones de porqué se fue" o "qué hice para que esto pasara"; (d) reconciliación $(\alpha=.69 ; .71)$, con oraciones como ...debe (pensar)... "en momentos que pasaron juntos", "en planear una solución" o "en reconquistarlo(a)"; y (e) fatalismo $(\alpha=.64 ; .64)$, con afirmaciones como ...debe (pensar)... "en el suicidio", "en lo malo de la relación" y "que la vida no tiene sentido".

Por otra parte, en la subescala afectiva se incluyen cuatro factores, a saber: (a) devaluación $(\alpha=.89 ; .90)$, con ítems como ...debe (sentir)... "que no valgo nada", "ganas de morirme" o "inseguridad"; (b) melancolía $(\alpha=.83 ; .83)$, con reactivos como ...debe (sentir)... "nostalgia", "tristeza" o "ganas de llorar"; (c) terrible $(\alpha=.83 ; .83)$, con afirmaciones como ...debe (sentir)... "feo", "mal" y "horrible"; $\mathrm{y}(\mathrm{d})$ resentimiento $(\alpha=.82 ; .83)$, con oraciones como ... debe (sentir)... "rencor", "rabia" y "odio".

Y, por último, en la subescala conductual se incluyen cuatro factores, a saber: (a) fortaleza $(\alpha=.91 ; .92)$, con ítems como ... debe (hacer)... "salir adelante", "tranquilizarse" o "superarlo"; (b) quedar en paz ( $\alpha=.72 ; .73)$, con oraciones como ... debe (hacer)... "buscar una reconciliación", "arreglar el problema" o "hablar con su ex respecto a lo que ocurrió"; (c) animarse $(\alpha=.69 ; .69)$, con reactivos como ...debe (hacer)... "no hacer tonterías", "no deprimirse" y "desahogarse"; y (d) buscar alternativas $(\alpha=.65 ; .65)$, con afirmaciones como ...debe (hacer)... "buscar otra pareja", "esperar a que llegue otra persona le ame" o "conocer a otras personas".

Escala de estilos de apego adulto (Márquez-Domínguez, Rivera-Aragón \& Reyes-Lagunes, 2009). Este instrumento, en su versión corta, consta de 18 reactivos en formato de respuesta tipo Likert con cinco grados de acuerdo o desacuerdo. Específicamente, se compone de tres factores: (a) apego evitativo $(\alpha=.94 ; .79)$, con enunciados como "me incomoda que mi pareja procure mucha cercanía", "me doy cuenta de que justo cuando mi pareja empieza a lograr algo de intimidad emocional conmigo, me alejo" o "preferiría no estar muy cercano a mi pareja"; (b) apego ansioso-ambivalente $(\alpha=.93 ; .78)$, con afirmaciones como "me preocupa que mi pareja no se interese tanto en mí como yo en ella", "necesito que mi pareja me confirme constantemente que me quiere" $\mathrm{y}$ "a veces siento que presiono a mi pareja a comprometerse $\mathrm{y}$ demostrarme que me quiere"; y (c) apego seguro $(\alpha=.90$; $.75)$, con reactivos como "pienso que la relación marcha 
bien", "tiendo a ser feliz" y "confío en que mi pareja me ama tanto como yo la amo a ella".

Escala de estrategias de rompimiento amoroso en abandonados (Jiménez-Rodríguez \& Sánchez-Aragón, 2017). Este tercer instrumento consta de 41 reactivos con formato de respuesta tipo Likert con cinco opciones de respuesta que indican grados de acuerdo o desacuerdo. Estos ítems se distribuyen en seis factores, a saber: (a) desinterés y apatía $(\alpha=.89 ; .89)$, con reactivos como "se mostraba indiferente"; "puso muchos pretextos para no vernos" o "era grosero y cortante"; (b) alejamiento ( $\alpha=.86 ; .87)$, con oraciones como "habló muy poco sobre sus actividades personales e intereses cuando conversábamos", "procuró no pedirme favores" o "evitó planear encuentros futuros cada vez que podía"; (c) cuidado compasivo y cortesía $(\alpha=.79 ; .80)$, con enunciados como "trató de reconfortarme a pesar de lo negativo de la situación", "fue gentil y directo al decirme lo que sentía", "buscó un lugar apropiado" o "me miró a los ojos y me tomó de las manos para decirme que la relación entre nosotros no debía continuar"; (d) pretextos $(\alpha=.78 ; .78)$, con reactivos como "provocó una discusión como una excusa para terminar la relación", "provocaba peleas" o "se volvió desagradable con la esperanza de que yo tomara la iniciativa para terminar la relación"; (e) justificación $(\alpha=.71 ; .71)$, que incluye ítems como "verbalmente me explicó sus razones para desear terminar la relación", "me expresó abiertamente su deseo de terminar la relación" o "fue totalmente sincero y honesto al exponer su deseo de terminar"; y (f) convencimiento $(\alpha=.70$; .70), con afirmaciones como "me dio muchas explicaciones para que comprendiera su posición", "hizo que pareciera que la relación era más costosa para mí diciéndome sus defectos" o "me propuso que tuviéramos otro tipo de relación, como ser amigos".

\section{Procedimiento}

Inicialmente, se asistió a centros educativos y laborales para la solicitar el consentimiento y participación voluntaria de personas a quienes su pareja les hubiera terminado una relación romántica en un periodo de menos de seis meses, que tuvieran escolaridad mínima de preparatoria, y que la duración de su relación hubiera sido de al menos un año. Una vez que aceptaron participar, se les entregó los instrumentos y se les brindó el tiempo suficiente para que pudieran responderlos - se tardaron aproximadamente media hora en hacerlosimultáneamente, se les aclaró las dudas que surgieran, y posteriormente se puso a su disposición los resultados. En la aplicación de los instrumentos se le garantizó a cada persona el anonimato y la confidencialidad de sus respuestas. Finalmente, una vez que se obtuvieron los datos, se procedió a dividir las respuestas de los participantes en dos grupos, el primero con una historia romántica positiva y el segundo con una historia negativa.

\section{Análisis de datos}

Para iniciar, se realizó la prueba Kolmogorov-Smirnov para determinar si los datos cumplían con el supuesto de normalidad, y como los datos no cumplieron los requisitos de distribución normal ni de dispersiones homogéneas, se procedió a identificar las correlaciones por medio de la prueba de Spearman. En todos los casos se fijó el nivel de significancia como $<.05$.

\section{Resultados}

Para responder al objetivo planteado — después de realizar la prueba de Kolmogorov-Smirnov y comprobar que las variables no cumplen en supuesto de normalidad; y tomando en cuenta que la selección de las pruebas estadísticas adecuadas y ajustadas al tipo de distribución real provoca una reducción del error tipo I (Schucany \& Ng, 2006)-, se procedió a establecer el coeficiente de correlación de Spearman (rho), uno para los participantes abandonados con una historia amorosa positiva y otro para los de historia amorosa negativa.

A continuación se presentan los resultados, divididos en dos apartados. En el primero se encuentran los análisis de la relación entre el tipo de apego y las PHSC con el duelo en las personas abandonadas con historia positiva, y en segundo, un análisis igual de las personas con historia romántica negativa.

En primer lugar, los hallazgos muestran que, tal como se observa en la Tabla 1, para los participantes abandonados (hombres o mujeres) con historia romántica positiva:

1. Cuando el participante cree que ante el duelo se debe sentir resentimiento, que todo es terrible, que debe animarse y ser optimista, además de que tiene un estilo de apego ansioso-ambivalente y que cree que se debe sentirse devaluado, entonces esta persona percibe que su expareja mostró un gran desinterés y que fue cortante y grosera como estrategia para terminar la relación.

2. Cuando el participante cree que hay que sentir resentimiento, que la situación es terrible, pero que hay algo de optimismo, y no cree en la reconciliación ante el rompimiento, esta persona percibe que su expareja se fue alejando poco a poco.

3. Cuando el participante cree que hay que reconciliarse y quedar en paz con la expareja, y además tiene un estilo de apego evitativo, entonces percibe que la expareja utilizó la estrategia de cuidado compasivo y cortesía.

4. Cuando el participante tiene un estilo de apego evitativo, ansioso o seguro, pero cree que se debe sentir 
resentimiento, devaluación o que el rompimiento es terrible, además de que cree que debe pensar de manera fatal o en los motivos de la separación, y no en la superación, aunque sí ser algo optimista y creer que se debe ser fuerte, de este modo, entonces, percibe que su expareja utilizó pretextos para terminar con la relación.

5. Cuando el participante cree que tiene otras alternativas y que debe ser optimista, entonces percibe que quien terminó la relación lo hizo justificándose.

6. Y, por último, cuando el participante cree que ante el duelo se debe pensar en que no hay que superar la situación, que debe ser fatalista y creer que se debe sentir devaluación y resentimiento, además de no mostrar fortaleza, sumado a que tiene un apego evitativo, entonces esta persona percibe que su expareja le explicó de manera sincera, honesta y abierta las razones por las cuales lo más indicado era terminar la relación.

Y, en segundo lugar, tal como se observa en la Tabla 2, en el caso de los participantes (hombres o mujeres) abandonados con una historia romántica negativa, se observa que:

1. En la medida en que el participante con el papel pasivo cree que ante el rompimiento se debe sentir terrible, así como devaluación, melancolía y resentimiento, además de que cree que se debe pensar en los motivos y tratar de quedar en paz, todo unido a tener un apego ansioso-ambivalente, entonces esta persona percibe que la estrategia usada por su expareja para finalizar la relación fue de desinterés y apatía.

2. Cuando el participante tiene apego ansioso-ambivalente y cree que tras el rompimiento tiene que sentirse devaluado, terrible y con resentimiento, además de pensar sobre los motivos que dieron término a la relación, entonces va a percibir que su expareja utilizó el alejamiento como estrategia para la ruptura.

3. En la medida en que se tiene un estilo de apego evitativo y se cree que tras el rompimiento hay que quedar en paz con el otro, de este modo se percibe que la expareja usó como estrategia el cuidado compasivo y la cortesía.

4. Cuando el participante cree que en el duelo se debe sentir desvalorización, resentimiento, terrible y melancolía, además de que se debe pensar en los motivos del quiebre y no creer que exista razón para sentirse bien, y a la vez tiene un apego ansioso-ambivalente o evitativo, entonces esta persona percibe que su rompimiento tuvo como base pretextos continuos, volviéndose desagradable y provocando peleas.

5. Por último, en la medida en que el participante tiene apego ansioso-ambivalente y cree que ante el duelo se debe sentir terrible y sin valor, además de pensar con optimismo

Tabla 1.

Relación entre los estilos de apego y las PHSC del duelo romántico con las estrategias de rompimiento en abandonados con historia romántica positiva

\begin{tabular}{|c|c|c|c|c|c|c|c|}
\hline & & $\begin{array}{c}\text { Desinterés } \\
\text { y apatía }\end{array}$ & Alejamiento & $\begin{array}{c}\text { Cuidado compasi- } \\
\text { vo y cortesía }\end{array}$ & Pretextos & Justificación & Convencimiento \\
\hline \multirow{4}{*}{$\begin{array}{l}\text { PHSC } \\
\text { Sentir }\end{array}$} & Devaluación & $.114^{*}$ & - & - & $.247^{* *}$ & - & $.163^{* *}$ \\
\hline & Melancolía & - & - & - & & - & - \\
\hline & Terrible & $.151^{* *}$ & $.129^{*}$ & - & $.153^{* *}$ & - & - \\
\hline & Resentimiento & $.188^{* *}$ & $.154^{* *}$ & - & $.291^{* *}$ & - & $.132^{*}$ \\
\hline \multirow{5}{*}{$\begin{array}{l}\text { PHSC } \\
\text { Pensar }\end{array}$} & Superación & - & - & - & $-.138^{* *}$ & - & $-.179^{* *}$ \\
\hline & Optimismo & $.115^{*}$ & $.105^{*}$ & - & $.105^{*}$ & $.114^{*}$ & - \\
\hline & Motivos & - & & - & $.155^{* *}$ & - & - \\
\hline & Reconciliación & - & $-.102^{*}$ & $.232^{* *}$ & & - & - \\
\hline & Fatalismo & - & - & - & $.254^{* *}$ & - & $.158^{* *}$ \\
\hline \multirow{4}{*}{$\begin{array}{l}\text { PHSC } \\
\text { Hacer }\end{array}$} & Fortaleza & - & - & - & $-.145^{* *}$ & - & $-.126^{*}$ \\
\hline & Quedar en paz & - & - & $.137^{* *}$ & - & - & - \\
\hline & Animarse & $.146^{* *}$ & - & - & - & - & - \\
\hline & Alternativas & - & - & - & - & $.133^{*}$ & - \\
\hline \multirow{3}{*}{$\begin{array}{c}\text { Estilos de } \\
\text { apego }\end{array}$} & Seguro & - & - & - & $-.203^{* *}$ & - & - \\
\hline & Ansioso-ambivalente & $.118^{*}$ & - & - & $.231^{* *}$ & - & - \\
\hline & Evitativo & - & - & $.184^{* *}$ & $.233^{* *}$ & - & $.171^{* *}$ \\
\hline
\end{tabular}

Nota. $* \mathrm{p}<.05 ; * * \mathrm{p}<.01 ; * * * \mathrm{p}<.001$. 
Tabla 2.

Relación entre los estilos de apego y los PHSC del duelo romántico con las estrategias de rompimiento en abandonados con historia romántica negativa

\begin{tabular}{|c|c|c|c|c|c|c|c|}
\hline & & $\begin{array}{c}\text { Desinterés } \\
\text { y apatía }\end{array}$ & Alejamiento & $\begin{array}{c}\text { Cuidado } \\
\text { compasivo y } \\
\text { cortesía }\end{array}$ & Pretextos & Justificación & Convencimiento \\
\hline \multirow{4}{*}{$\begin{array}{l}\text { PHSC } \\
\text { Sentir }\end{array}$} & Devaluación & $.231^{* *}$ & $.170^{*}$ & - & $.325^{* *}$ & $.145^{*}$ & $.170^{*}$ \\
\hline & Melancolía & $.221^{* *}$ & - & - & $.155^{*}$ & - & - \\
\hline & Terrible & $.239^{* *}$ & $.146^{*}$ & - & $.270^{* *}$ & - & $.214^{* *}$ \\
\hline & Resentimiento & $.149^{*}$ & .106 & - & $.274^{* *}$ & - & $.136^{*}$ \\
\hline \multirow{5}{*}{$\begin{array}{l}\text { PHSC } \\
\text { Pensar }\end{array}$} & Superación & - & - & - & - & - & - \\
\hline & Optimismo & - & - & - & - & - & $.155^{*}$ \\
\hline & Motivos & $.170^{*}$ & $.181^{* *}$ & - & $.212^{* *}$ & - & - \\
\hline & Reconciliación & - & - & - & - & - & - \\
\hline & Fatalismo & - & - & - & $.319^{* *}$ & - & - \\
\hline \multirow{4}{*}{$\begin{array}{l}\text { PHSC } \\
\text { Hacer }\end{array}$} & Fortaleza & - & - & - & - & - & - \\
\hline & Quedar en paz & $.142^{*}$ & - & $.150^{*}$ & - & - & - \\
\hline & Animarse & - & - & - & $-.144^{*}$ & - & - \\
\hline & Alternativas & - & - & - & - & - & $.165^{*}$ \\
\hline \multirow{3}{*}{$\begin{array}{c}\text { Estilos } \\
\text { de apego }\end{array}$} & Seguro & - & - & - & $-.147^{*}$ & - & - \\
\hline & Ansioso-ambivalente & $.330^{* *}$ & $.243^{* *}$ & - & $.299^{* *}$ & - & $.307^{* *}$ \\
\hline & Evitativo & - & - & $.157^{*}$ & $.164^{*}$ & - & $.165^{*}$ \\
\hline
\end{tabular}

Nota $* \mathrm{p}<.05 ; * * \mathrm{p}<.01 ; * * \mathrm{p}<.001$.

y que pueden existir alternativas en el futuro, de este modo percibe que su expareja trató de convencerla por todos los medios para que creyera que terminar era la mejor opción.

\section{Discusión}

La vinculación con el otro es una necesidad que se tiene desde el momento en que se nace y se conserva a lo largo de la vida; después, cuando se crece, la figura de apego ya no es la madre, sino que entonces se elige con quién se establece un vínculo, el cual, entre otros, puede ser del tipo de relación romántica, ya que a medida que pasan los años se va volviendo el centro de la vida de cada persona. Pero cuando estas relaciones se terminan y se tiene que enfrentar el rompimiento amoroso aparece un problema importante, ya que con esto se pasa por un proceso sumamente doloroso que, dada su complejidad y por las variables que involucra, exige su estudio por parte de la psicología. Teniendo esto en cuenta, el propósito de la presente investigación fue identificar la relación entre las PHSC del duelo romántico y los estilos de apego con las estrategias de rompimiento percibidas por los abandonados con una historia romántica positiva o negativa.
PHSC, estilos de apego y percepción de estrategias de rompimiento en participantes con historia romántica positiva

Los hallazgos muestran, para los participantes abandonados con historia romántica positiva, que en la medida en que el participante cree que ante el duelo se debe sentir resentimiento, que todo es terrible, pero que debe animarse y ser optimista, sumado a tener un estilo de apego ansiosoambivalente y creer que se debe sentir devaluado, entonces esta persona percibe que su expareja mostró gran desinterés, y que incluso fue cortante y grosera como estrategia para terminar la relación. Al respecto, Merino-Ramírez (2014) encontró que el resentimiento no tiene ninguna utilidad en este proceso de duelo, sino que, por el contrario, frena todo avance; además de que si las personas se enfocan en lo positivo de la injusticia, ello les servirá para ver a la expareja de manera distinta, y así poder liberarse del odio y del resentimiento, $\mathrm{y}$ dar paso a fases de mayor aceptación, optimismo y positividad (Sánchez-Aragón \& Retana-Franco, 2013). Si a esto se le suma que la personas tengan un apego ansioso-ambivalente - es decir, que perciban poco acceso a la persona y una gran necesidad de tenerla (Mikulincer \& Shaver, 2003) - el que sean dejados por la pareja reafirma la ansiedad experimentada por no tener a la persona en la medida en que la necesitan. 
Por otra parte, cuando el participante cree que hay que sentir resentimiento y que la situación es terrible, pero también algo de optimismo, y no cree en la reconciliación ante el rompimiento, esta persona percibe que su expareja se fue alejando poco a poco. En este caso, el hecho de que las personas consideren que la manera en que terminaron con ellos fue por medio de un alejamiento de la relación y de dejar pasar aspectos que antes eran relevantes, termina favoreciendo el rencor, y esto, aunado a la creencia de que se debe sentir terrible por estar en dicha situación, tener poco optimismo y no creer en la reconciliación, hace que el proceso por el que están pasando sea más duro y desesperanzador. Esto confirma lo mencionado por Caruso (2003) y Fisher (2005) con respecto a que el rompimiento amoroso es una de las experiencias más dolorosas para el ser humano, que varía en intensidad, que favorece la afectividad negativa (véase también Rosales-Sarabia, Rivera-Aragón \& García-Méndez, 2015) y que, si no se maneja adecuadamente, puede llegar a tener consecuencias muy graves que pueden causar ansiedad y depresión. Cabe mencionar que el ligero optimismo que se observa puede ser un efecto de la reevaluación objetiva que hace la persona de la situación, reevaluación que le permite pensar que las cosas pasan por algo y que vendrán cosas mejores en las relaciones; este viso de positividad le permite al individuo minimizar las experiencias negativas (Páez, Bilbao \& Javaloy, 2008).

También, cuando la persona abandonada percibe que su expareja decidió terminar la relación de manera directa y expresando una verdadera preocupación por lo que pudiera sentir, esta persona puede creer que hay posibilidades de reconciliación, aunque esto no sea así. De este modo, pareciera que en el pensamiento de los abandonados queda en un impasse que brinda un buen recuerdo de la relación que ya no puede seguir, pero, al mismo tiempo, esa cortesía y cuidado puede interpretarse como una "puerta abierta hacia un regreso". Estos resultados son consistentes con lo reportado por Sprecher et al. (2010), en donde se menciona que cuando se utiliza esta estrategia, las personas tienen menor impacto negativo posterior, aunque no una sensación determinante de rompimiento.

En otro caso, los participantes que percibieron que la relación fue terminada por su expareja con comunicación indirecta - es decir, con el uso de excusas y evasivas para salir de la relación - tienden a creer que ante el duelo una persona debe sentirse terrible, devaluación o resentimiento, y pensar en sus razones y con fatalidad; sin embargo, a pesar de creer que "hay que ser fuerte", dichas creencias dificultan la superación. Estos resultados concuerdan con lo encontrado por Metts et al. (1989) y Sprecher et al. (2010), en donde se menciona que dicha manera de rompimiento se asocia con resultados mas negativos posteriores a la ruptura, como el hecho de que las personas piensen que tienen que sentir cosas muy negativas hacia sus exparejas. Además, si esta forma de ruptura es acompañada de un apego evitativo, habrá una mayor probabilidad de reacciones de hostilidad al sentir el abandonado, sobre todo cuando se habían bajado las barreras para que el otro entrara en su vida (Dutton, Starzomski \& Van Ginkel, 1995). Adicional a esto, Merino-Ramírez (2014) menciona que el creer poco en la posibilidad de superar un rompimiento, aunado a la creencia de que se posee fortaleza y de ser optimista representa un primer acercamiento a la aceptación de la pérdida de la pareja, sin embargo, en ocasiones no se tiene la fortaleza necesaria para reencontrarse a sí mismo y reconocer que la vida es mucho más que todo lo que pareciera estar perdido, lo cual da pauta a lo que se conoce como pseudoaceptación (Sánchez-Aragón \& Martínez-Cruz, 2014).

Por otra parte, también se encontró que cuando el participante cree que ante el rompimiento amoroso se debe pensar en que hay alternativas y que debe ser optimista, este percibió que el rompimiento se dio mediante la justificación - es decir, una explicación sincera y abierta de las razones por las cuales deseaba terminar la relación-. Este caso muestra un escenario distinto, donde la persona asimiló el suceso de una manera menos negativa, todo gracias a estas creencias y, posiblemente, a otros elementos propios de la relación, como pueden ser el involucramiento emocional o la duración de la relación (Sánchez-Aragón \& Martínez-Cruz, 2014).

Finalmente, en el último caso encontrado, cuando el participante cree que ante el duelo se debe pensar en que no hay que superar la situación, que hay que ser fatalista y creer que se debe sentir devaluación, resentimiento y no mostrar fortaleza, esto sumado a tener un apego evitativo, entonces dicha persona percibe que, cuando su relación terminó, su expareja intentó convencerle de que lo mejor era terminar la relación y abrir la posibilidad hacia una amistad. Estos resultados apoyan lo mencionado por MerinoRamírez (2014), en donde se afirma que dicha estrategia de rompimiento le permite a la persona con rol pasivo aceptar y agradecer la experiencia vivida - por muy dolorosa que esta sea-, otorgarle un justo valor a la experiencia, salir fortalecida y permitirle que aproveche la experiencia adquirida, aprenda de la situación y se sienta preparada para siguientes retos. No obstante, en este escenario, las creencias de la persona son negativas respecto al sentir y al pensar, lo cual refleja su inconformidad ante el evento, y esto puede vincularse con un estilo de apego evitativo, puesto que en este estilo se enfatiza una duda continua sobre el amor y sobre la importancia de relacionarse positivamente con otra persona. De este modo, el tipo de apego mismo se ve reforzado con creencias negativas y desalentadoras ante 
el rompimiento, lo cual promueve que se le quite el valor positivo al vínculo amoroso perdido (Bartholomew, 1990; Bartholomew \& Horowitz, 1991; Collins \& Read, 1994; Feeney, Noller \& Callan, 1994).

PHSC, estilos de apego y percepción de estrategias de rompimiento en participantes con historia romántica negativa

Ahora bien, en el caso de haber vivido una historia de amor evaluada con tintes negativos, los resultados tienen una clara diferenciación a cuando se perciben de manera positiva. Así, en primer lugar, cuando se percibe que la estrategia que se utilizó para terminar la relación fue el desinterés y la apatía, o bien el alejamiento, la persona con rol pasivo tiene creencias que refuerzan la experiencia de sentirse muy mal, desvalorizado, triste, con odio y desprecio por la expareja (Metts et al., 1989; Perilloux \& Buss, 2008). Ante dichos sentimientos, esta se pregunta qué fue lo que pudo haber hecho para que sucediera el rompimiento, pero se da cuenta de que ya no puede hacer nada para solucionar lo ocurrido. Si estas características se suman a un apego ansioso-ambivalente -en el que la persona duda y es insegura-, se generará una sensación de inquietud permanente, en la que se manifiestan conductas de enojo y protesta (Tzeng, 1992). Al respecto, Sbarra (2006) reporta que en los participantes este estilo de apego es más difícil superar la tristeza de la pérdida y aceptar que la relación había terminado.

Por otra parte, cuando se utiliza la cortesía y el cuidado de los sentimientos de la persona con el rol pasivo, se encuentra concordancia con lo reportado por Sprecher et al. (2010) con respecto a que, al utilizar estrategias más positivas, existirá menor impacto y angustia en la otra persona, y esto se ve reflejado cuando las personas consideran que después de la ruptura deben de dejar las cosas así. Al respecto, Merino-Ramírez (2014) y Retana-Franco, Silva-Moctezuma y Sánchez-Aragón (2018) indican que, ante eventos como este, atributos como la resiliencia, la autoconfianza, la estructura y la fortaleza juegan un papel muy importante en la forma de reaccionar. En este caso, los participantes que además cuentan con un estilo de apego evitativo tienden a "resolver mejor" la pérdida, pues tienen una tendencia a la independencia y a la distancia interpersonal. Esto puede resultar en que la persona refuerce el no concentrarse en la pérdida, sino en las ganancias del rompimiento (Urra, 2010).

Tal como reporta Sprecher et al. (2010) con respecto a que las estrategias negativas traerán mayor sufrimiento, en este trabajo se encuentra que cuando se utilizan excusas y justificaciones para salir de la relación, las personas abandonadas consideran que lo que se debe pensar es que todo será malo en su vida, y que debe preguntarse por las razones de lo sucedido. Aunado a ello, tener un apego ansioso-ambivalente favorece la experiencia de descontento, desesperanza y tensión ante el rompimiento, ya que estas personas expresan que necesitan a la pareja para sentirse completos (Melero \& Cantero, 2008).

Por ultimo, cuando la persona con rol pasivo percibe que la expareja dijo de manera clara los inconvenientes para continuar la relación, es decir, que le convenció de las razones del rompimiento, esto se relacionó con creer que se debe sentir terrible, muy mal consigo mismo y con el rencor hacia la expareja. Con respecto a esto, en otros estudios se ha encontrado que este tipo de estrategia percibida contribuye a que la persona - a pesar de vivir una situación devastadora (Caruso, 2003; Sánchez-Aragón \& Retana-Franco, 2013) — tenga un impacto emocional más atenuado (Metts et al., 1989; Sprecher et al., 2010). Esta estrategia es percibida de esta forma, ya que, aunque reconocen lo difícil de la situación en términos emocionales, también perciben que algo mejor vendrá después y que encontrarán a otra persona pronto (Larraburu, 2010). Esto último incrementa si se tiene un apego ansioso-ambivalente o evitativo, ya que se generará una de inquietud permanente, en donde se ve el enojo y el sentirse muy mal, pero al mismo tiempo se siente alivio de que esto terminará en algún momento (Tzeng, 1992).

En conclusión, tras el análisis de los resultados se puede decir que tanto la cultura como el estilo de apego tienen un impacto importante en las estrategias de rompimiento percibidas. Aunque no se observa un patrón constante, sí se puede decir que el hecho de que el participante con historia romántica positiva perciba que su relación terminó por pretextos de su expareja es porque sus creencias socioculturales son de devaluación, resentimiento y fatalismo, y porque su estilo de apego es evitativo o ansioso-ambivalente. Por otra parte, las personas abandonadas con historia romántica negativa perciben que su relación terminó por pretextos y desinterés por parte de su expareja, sobre todo cuando tienen creencias de devaluación, melancolía, de sentirse terrible, y cuando sienten resentimiento y fatalismo. Aquí, el estilo ansioso-ambivalente mostró un efecto sostenido en el que, al parecer, se percibe que el rompimiento se debió a estrategias negativas.

Es importante mencionar que las correlaciones existen en casi todos los casos, aunque con una magnitud baja, por lo que se recomienda seguir trabajando con esta temática, sobre todo para identificar el impacto que tiene el rompimiento no solo en la persona que ejerce un rol pasivo, sino también en la que se considera como agente, y evaluar las maneras de pensar, sentir y actuar tras la ruptura dependiendo de la estrategia de rompimiento utilizada. Adicional a 
esto, en general, los resultados de la presente investigación confirmaron lo encontrado en investigaciones precedentes, pero también se encuentran datos novedosos que quizá solo aparezcan en la cultura mexicana; para confirmar esta hipótesis se necesita realizar una investigación transcultural que permita identificar cómo se comporta el fenómeno en otras culturas.

Aún queda mucho por hacer, pero con la presente investigación se busca mostrar una visión adicional ante un fenómeno tan complejo, ya que en la mayoría de las investigaciones se analizan las diferentes etapas amorosas, pero se deja de lado lo que ocurre una vez que se termina la relación, así como los impactos que tiene dicho rompimiento en los miembros de la pareja.

\section{Referencias}

Agnew, C. R. (2000). Cognitive interdependence and the experience of relationship loss. En J. H. Harvey \& E. D. Miller (eds.), Loss and trauma: General and close relationship perspectives (vol. 3, pp.163-188). Londres: Jessica Kingsley Publishers.

Ainsworth, M. D. (1969). Objects relations, dependency and attachment: A theoretical reviews of the infant-mother relationship. Child Development, 40(4), 969-1025. doi: http:// dx.doi.org/10.2307/1127008

Banks, S. P., Altendorf, D. M., Greene, J. O., \& Cody, M. J. (1987). An examination of relationship disengagement: Perceptions, breakup strategies, and outcomes. Western Journal of Speech Communication, 51(1), 19-41. doi: http:// dx.doi.org/10.1080/10570318709374250

Bartholomew, K. (1990). Avoidance of intimacy: An attachment perspective. Journal of Social and Personal relationships, $\quad 7(2), \quad$ 147-178. doi: http://dx.doi. org/10.1177/0265407590072001

Bartholomew, K., \& Horowitz, L. M. (1991). Attachment styles among young adults: A test of a four-category model. Journal of Personality and Social Psychology, 61(2), 226244. doi: http://dx.doi.org/10.1037/0022-3514.61.2.226

Becerril, D. (2001). Relaciones de pareja. Revista de Educación, 325, 49-56. Recuperado de https://sede.educacion.gob.es/ publiventa/descarga.action?f_codigo_agc=9962_19

Bowlby, J. (1973). Attachment and loss: attachment. Nueva York: Basic Books.

Caruso, I. (2003). La separación de los amantes: Una fenomenología de la muerte. México: Siglo XXI.

Collins, N. R., \& Read, S. J. (1994). Cognitive representations of attachment: The structure and function of working models. En K. Bartholomew \& D. Perlman (eds.), Advances in personal relationships (pp. 53-90). Londres: Jessica Kingsley Publishers.
Collins, T. J., \& Gillath. O. (2012). Attachment, breakup strategies, and associated outcomes: The effects of security enhancement on the selection of breakup strategies. Journal of Research in Personality, 46(1), 210-222. doi: 10.1016/j. jrp.2012.01.008

Davis, D., Shaver, P. R., \& Vernon, M. L. (2003). Physical, emotional, and behavioral reactions to breaking up: The roles of gender, age, emotional involvement, and attachment style. Personality and Social Psychology Bulletin, 29(7), 871-884. doi: 10.1177/0146167203029007006

Díaz-Loving, R., \& Sánchez-Aragón, R. (1998). Premisas y Normatividad en las Parejas Mexicanas. La Psicología Social en México, VII, 129-136.

Díaz-Guerrero, R. (2003). Psicología del mexicano: Descubrimiento de la etnopsicología. México: Trillas.

Dutton, D. G., Starzomski, A., \& Van Ginkel, C. (1995). The role of shame and guilt in the intergeneracional transmisión of abusiveness. Violence and Victims, 10(2), 121-131. doi: 10.1891/0886-6708.10.2.121

Dutton, L. B., \& Winstead, B. (2006). Predicting unwanted pursuit: Attachment, relationship satisfaction, relationship alternatives, and break-up distress. Journal of Social and Personal Relationships, 23(4), 565-586. doi: 10.1177/0265407506065984

Feeney, J. A., \& Noller, P. (1992). Attachment style and romantic love: Relationship dissolution. Australian Journal of Psychology, 44(2),69-74. doi: 10.1080/00049539208260145

Feeney, J. A., Noller, P., \& Callan, V. J. (1994). Attachment style, communication and satisfaction in the early years of marriage. En D. Perlman \& K. Bartholomew (eds.), Advances in personal relationships (pp. 269-308). Londres: Jessica Kingsley Publishers.

Feldberg, R., \& Kohen, J. A. (1980). Family life in an antifamily setting: A critique of marriage and divorce. En J. M. Henslin (ed.), Marriage and family in a changing society (pp. 415-427). Nueva York: The Free Press.

Fisher, H. (2005). Por qué amamos. México: Santillana.

Fuller, T. L., \& Finchman, F. D. (1995). Attachment style in married couples: Relation to current marital functioning, stability over time, and method of assessment. Personal Relationship, 2(1), 17-34. doi: https://doi. org/10.1111/j.1475-6811.1995.tb00075.x

Guttmann, J. (1993). Divorce in Psychosocial Perspective: Theory and Research. New Jersey: Lawrence Erlbaum Associates, Inc., Publishiers.

Hazan, C., \& Shaver, P. (1987). Conceptualizing romantic love as an attachment process. Journal of Personality and Social Psychology, 52(3), 511-524. doi: 10.1037/0022-3514.52.3.511

Jaramillo-Bustamante, M. E. (2015). Estrategias de afrontamiento tras una ruptura de relaciones afectivas en jóvenes adultos de la carrera de ciencias de la comunicación social del área de la educación, arte y comunicación, de la 
Universidad Nacional de Loja (tesis de licenciatura no publicada). Universidad Nacional De Loja, Ecuador.

Jiménez-Rodríguez, B., \& Sánchez-Aragón, R. (2017, noviembre). Identificando las Estrategias de Rompimiento Amoroso en Agentes y Pasivos. Enviado a evaluación a la Revista Costarricense de Psicología.

Kerlinger, F. N., \& Lee, H. B. (2002). Investigación del comportamiento. Métodos de investigación en ciencias sociales (4. ${ }^{\mathrm{a}}$ ed.). México: McGraw-Hill.

Larraburu, I. (2010). Superar el desamor. La vanguardia. Recuperado de http://www.isabel- larraburu.com/articulos/ pareja/121-superar-el-desamor-.pdf

Le, B., Dove, L. N., Agnew, C., Korn, M. S., \& Mutso, A. A. (2010). Predicting non-marital romantic relationship dissolution: A meta-analytic synthesis. Personal Relationships, 17(3), 377-390. doi: 10.1111/j.1475-6911.2010.01285.x

Lewandowski, G. W. Jr., \& Bizzoco, N. (2007). Addition through subtraction: Positive outcomes following the loss of a dating relationship. Journal of Positive Psychology, 2(2), 40-54. doi: 10.1080/17439760601069234

Locker, L. J., McIntosh, W. D., Hackney, A. A., Wilson, J. H., \& Wiegand, K. E. (2010). The breakup of Romantic Relationships: Situational Predictors of Perception of Recovery. North American Journal of Psychology, 12(3), 565-578. Recuperado de http://digitalcommons.georgiasouthern.edu/psych-facpubs/97

Márquez-Domínguez, F., Rivera-Aragón, S., \& Reyes-Lagunes, I. (2009). Desarrollo de una escala de estilos de apego adulto para la población mexicana. Revista Interamericana de Psicología, 28(2), 9-30. Recuperado de http://www.redalyc. org/articulo.oa? $\mathrm{id}=459645444002$

Melero, R., \& Cantero, M. J., (2008). Los estilos afectivos en la población española: un cuestionario de evaluación del apego adulto. Clínica y Salud, 19(1), 83-100. Recuperado de http://scielo.isciii.es/scielo.php?script=sci_arttext\&pid $=$ S1130-52742008000100004

Merino-Ramírez, P. (2014). Duelo por abandono de pareja (tesis de maestría no publica). Asociación Mexicana de Tanatología A. C., México.

Metts, S., Cupach, W. R., \& Bejlovec, R. A. (1989). "I love you too much to ever start liking you": Redefining romantic relationships. Journal of Social and Personal Relationships, 6(3), 259-274. doi: http://dx.doi. org/10.1177/0265407589063002

Mikulincer, M., \& Shaver, P. (2003). The attachment behavioral system in adulthood: activation, psychodynamics, and interpersonal processes. Advances in Experimental Social Psychology, 35, 53-152. doi: 10.1016/ S0065-2601(03)01002-5

Páez, D., Bilbao, M. A., \& Javaloy, F. (2008). Del trauma a la felicidad. Hechos vitales y procesos socio cognitivos de crecimiento personal e interpersonal. En M. M. Casullo (ed.), Prácticas de Psicología Positiva (pp. 139-202). Argentina: Si libro.
Perilloux, C., \& Buss, D. M. (2008) Breaking up romantic relationships. Costs experienced, and coping strategies deployed. Evolutionary Psychology, 6(1), 164-181. doi: https://doi.org/10.1177/147470490800600119

Rage, E. (2002). La pareja: elección, problemática y desarrollo. México: Plaza y Valdés.

Remshard, R. (1998). Adult Attachment Styles, Love styles, sexual Attitudes and sexual Behaviors of college students. Dissertation Abstracts International: Section B: The Sciences and Engineering, 59(10-B), 5622.

Retana-Franco, B. E., Silva-Moctezuma, D., \& SánchezAragón, R. (2018). Resiliencia y Consecuencias del Rompimiento Amoroso: Medición y Relación en Pasivos. En R. Díaz Loving, L. I. Reyes Lagunes \& F. López Rosales (eds.), La Psicología Social en México (vol. XVII, pp. 167-183). Monterrey: Asociación Mexicana de Psicología Social.

Rosales-Sarabia, R. M., Rivera-Aragón, S., \& García-Méndez, M. (2015). Perdón, Resentimiento y estilos de apego en la relación de pareja: un estudio correlacional. Memorias de XLII Congreso Nacional de Psicología. Recuperado de https://www.researchgate.net/publication/317056846_ Perdon_Resentimiento_y_Estilos_de_Apego_en_la Relacion_de_Pareja_Un_Estudio_Correlacional

Sánchez-Aragón, R. (2018). La socio-cultura del duelo romántico: medición de sus premisas. Revista Científica da Universidade do Mindelo, 5(1), 75-91.

Sánchez-Aragón, R., \& Martínez-Cruz, R. (2014). Causas y Caracterización de las Etapas del Duelo Romántico. Acta de Investigación Psicológica, 10(1), 1329-1343. Recuperado de http://www.scielo.org.mx/scielo.php?script=sci_arttext \&pid $=$ S2007-48322014000100002

Sánchez-Aragón, R., \& Retana-Franco, B. E. (2013). Evaluación Tridimensional del Duelo Amoroso en México. Revista Iberoamericana de Diagnóstico y Evaluación Psicológica, 36(2), 49-69. Recuperado de http://www.aidep.org/03_ri dep/R36/Art.\%203.pdf

Sbarra, D. A. (2006). Predicting the onset of emotional recovery following non-marital relationship dissolution: Survival analyses of sadness and anger. Personality and Social Psychology Bulletin, 32(3), 298-312. doi: 10.1177/0146167205280913

Schachner, D. A., \& Shaver, P. R. (2002). Attachment style and human mate poaching. New Review of Social Psychology, 1, 122-129. Recuperado de https://www.researchgate.net/ publication/281267929_Attachment_style_and_human mate_poaching

Schucany, W. R., \& Ng, H. K. (2006). Preliminary goodness-offit tests for normality do not validate the one-sample Student t. Communications in Statistics, Theory and Methods, 35(12), 2275-2286. doi: 10.1080/03610920600853308

Simpson, J. A. (1990). Influence of attachment styles on romantic relationships. Journal of Personality and 
Social Psychology, 59(5), 971-980. doi: http://dx.doi. org/10.1037/0022-3514.59.5.971

Sprecher, S., Zimmerman, C., \& Abrahams, E. M. (2010).

Choosing compassionate strategies to end a relationship: Effects of compassionate love for partner and the reason for the breakup. Social Psychology, 41(2), 66-75. Recuperado de https://pdfs.semanticscholar.org/a70f/49dcfe6a546a836 9df4bcb27efd32cd4a151.pdf

Tashiro, T., \& Frazier, P. (2003). "I'll never be in a relationship like that again": Personal growth following romantic relationship breakups. Personal Relationships, 10(1), 113-128. doi: http://dx.doi.org/10.1111/1475-6811.00039
Tzeng, O. (1992). Theories of Love Development, Maintenance, and Dissolution: Octagonal Cycle and Differential Perspectives. Nueva York: Praeger.

Urra, J. (2010). Estudio sobre fortalezas para afrontar las adversidades de la vida. Recuperado de http://www.javierurra. com/files/FortalezasParaAfrontarLasAdversidadesDeLa Vida.pdf

Waller, K. L. (2008). Trait self-esteem moderates the effect of initiator status on emotional and cognitive responses to romantic relationship dissolution. Dissertation Abstracts International, 69, 19-77. Recuperado de https://qspace. library.queensu.ca/bitstream/handle/1974/446/Waller_ Katherine_L_200707_PhD.pdf? sequence $=1 \&$ isAllowed $=\mathrm{y}$ 